\title{
ACTIVATION OF THE PROTOCOL OF MASSIVE HEMORRHAGE IN A PEDIATRIC HOSPITAL. RETROSPECTIVE ANALYSIS OF TWO YEARS
}

Pérez Ferrer A.,Viejo Llorente A., Navarro Suay R., Latorre J.

La Paz University Hospital, Dept of Anaesthesiology \& Intensive Care, Madrid, Spain

\section{Background:}

- The pediatric patient has a limited adaptation to hypovolemia, which is the origin of $12 \%$ of cardiorespiratory arrest in children.

- It is essential to develop protocols that facilitate the unification of criteria and communication between services for give a rapid response to a critical situation such as massive hemorrhage.

- We present the protocol of massive hemorrhage of the La Paz Children's Hospital and the analysis of its activation in a period of two years.

\section{Method:}

- The hospital's protocol of massive hemorrhage based on:

improved communication with the blood bank the use of a hemostatic resuscitation protocol

Protocolo de HM del Hospital - Formularios de petición de sangre: NO FIABLE Infantil La Paz

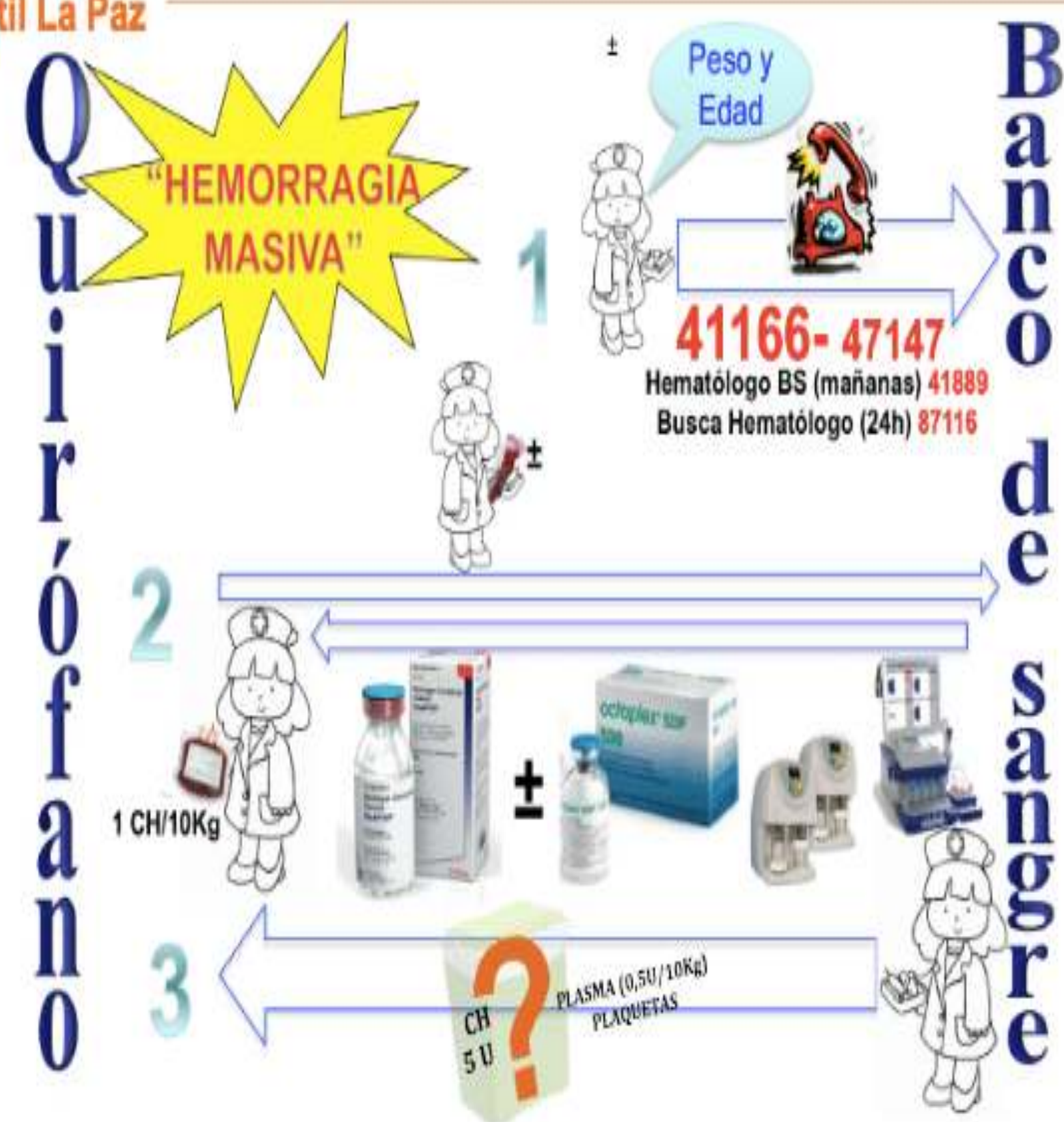

- A retrospective descriptive analysis of its activation between 2013 and 2014.

- Data were collected on the number of patients per year and the number of concentrated red blood cells.

\section{Discussion:}

- A total of 9 activations with a maximum of 19 units and a minimum of 4 .

\begin{tabular}{|c|c|c|c|} 
& Activations & Mean RBC & SD \\
\hline 2013 & 5 & 7.6 & 6.4 \\
\hline 2014 & 4 & 6 & 1.6 \\
\hline
\end{tabular}

- The proportion of RBC is higher than that of other blood components.

\section{Conclusion:}

* Every hospital must have a protocol of massive hemorrhage adapted to its characteristics that allows a coordinated and rapid response to correct and to avoid, as much as possible, hypovolemia and coagulopathy. 